\title{
Educación costarricense: relación entre los programas de Matemáticas y la política curricular
}

| Costa Rican Education: Relationship between Mathematics Programs and Curricular Policy |

\author{
Marianela Zumbado-Castro \\ mazumbado@uned.ac.cr \\ Universidad Estatal a Distancia \\ Costa Rica
}

Recibido: 18 Marzo 2020

Aceptado: 30 Agosto 2020

\begin{abstract}
Resumen. El artículo tiene como objetivo evidenciar la relación existente entre la metodología propuesta en los programas de Matemáticas y las dimensiones de la política curricular que están vigentes en Costa Rica. Para lograr este objetivo se plantean tres secciones. La primera consiste en un acercamiento a la estrategia metodológica planteada en los programas de matemáticas aprobados por el Consejo Superior de Educación (CSE) en el año 2012, incluyendo un ejemplo práctico de la primera etapa denominada "El aprendizaje del conocimiento". En segundo lugar, se presenta una descripción de las dimensiones de la política curricular aprobada por el CSE en el año 2016 y, finalmente, la tercera sección muestra como se relacionan los programas de Matemáticas con tales dimensiones. Como parte de las consideraciones finales, se sugiere la importancia de que el docente comprenda cómo se relacionan la propuesta metodológica vigente para la enseñanza y el aprendizaje de las Matemáticas con las dimensiones de la política curricular, para potenciar, a través de los momentos de la clase, el logro de las expectativas del sistema educativo.
\end{abstract}

Palabras clave: Enseñanza de las matemáticas, educación secundaria, política curricular, estrategia de enseñanza, resolución de problemas, educación costarricense.

Abstract. The objective of the article is to show the relationship between the methodology proposed in the Mathematics programs and the dimensions of the curricular policy that are in force in Costa Rica. To achieve this objective, three sections are proposed. The first consists of an approach to the methodological strategy proposed in the mathematics programs approved by the Higher Council for Education (CSE) in 2012, including a practical example of the first stage called "Learning knowledge". Secondly, a description of the dimensions of the curricular policy approved by the CSE in 2016 is presented and, finally, the third section shows how the Mathematics programs are related to these dimensions. As part of the final considerations, it is suggested that the teacher understand how the current methodological proposal for teaching and learning Mathematics is related to the dimensions of curricular policy, to enhance, through the moments of class, achieving the expectations of the educational system.

KeyWords: Mathematics education, secondary education, curricular policy, teaching strategies, problem solving, Costa Rican education. 


\subsection{Introducción}

Por casi veinte años estuvo vigente la "Política educativa hacia el Siglo XXI", la que fue considerada como la primera estrategia educativa a largo plazo en Costa Rica e incluyó la ampliación del curso lectivo a 200 días, la informática educativa en secundaria y un segundo idioma en la primaria. Además, implicó reformas constitucionales para establecer la obligatoriedad de la educación preescolar y la educación general básica hasta el noveno año, asimismo, la apertura de programas de apoyo económico para los estudiantes, entre otras acciones (Chavarría, 2010; Jiménez, 2014).

En 2016, el Consejo Superior de Educación (CSE) aprueba una nueva política curricular denominada "Educar para una Nueva Ciudadanía", la cual establece las aspiraciones del sistema educativo costarricense a través de cuatro dimensiones que promueven la formación de personas integrales.

Al considerar que en 2012 entraron en vigencia los programas de Matemáticas y la política curricular fue aprobada en 2016, es válido plantear cuestionamientos como los siguientes: ¿cuál es la relación entre los programas y la política? o ¿cuáles implicaciones tiene para el docente de Matemáticas la existencia o no de esa relación?

Con el objetivo de reflexionar sobre estas preguntas, se plantean tres secciones. En la primera se analiza qué proponen los programas de Matemáticas, cuál es su principal estrategia metodológica y, cómo se lleva a la práctica. En la segunda, se identifican las dimensiones de la política curricular. En la tercera, se establece la relación que existe entre ambas, para finalmente, reflexionar sobre las posibles implicaciones en las aulas.

\subsection{Programas de matemáticas y estrategia metodológica}

La resolución de problemas para la enseñanza y aprendizaje de las Matemáticas, ha estado presente en el ámbito educativo desde los años 40, con las ideas precursoras de Pólya (Barrantes, 2006; Zumbado, Espinoza, Espinoza, González y Ramírez, 2008). Por tanto, no es precisamente una novedad.

Lo que si resulta novedoso, es que en los programas de Matemáticas aprobados por el CSE en el año 2012, se asumiera la resolución de problemas, como estrategia metodológica principal, integrando ideas del constructivismo, el socioculturalismo, el pragmatismo realista y algunas tendencias didácticas asociadas con (1) las nociones que utiliza la Organización para la Cooperación y el Desarrollo Económico (OCDE) en las pruebas estandarizadas; (2) las ideas de Freudenthal sobre las Matemáticas realistas, (3) el modelo de la clase japonesa; (4) elementos provenientes de la escuela de la didáctica francesa (Zumbado-Castro, 2019). Esto generó una organización distinta para las lecciones que supuso dos etapas claramente diferenciadas: la primera enfatiza "el aprendizaje de conocimientos" y la segunda promueve "la movilización y aplicación de los conocimientos" (MEP, 2012, p. 41). A continuación, se describe cada una.

\section{Primera etapa: el aprendizaje de conocimientos}

La primera etapa corresponde al espacio de la clase para propiciar la adquisición del conocimiento. Se recomienda esta etapa para iniciar un tema utilizando una, dos o tres lecciones consecutivas preferiblemente. A su vez, el MEP (2012) propone descomponerla en cuatro momentos para realizar el abordaje del nuevo conocimiento.

En el primero de los momentos se hace la propuesta de un problema, el segundo es un espacio de tiempo para permitir el trabajo de los estudiantes en la tarea asignada; en el tercero, denominado "discusión interactiva y comunicativa", se debe generar un espacio de conversación para propiciar la interacción, el análisis y la reflexión por parte de los estudiantes sobre el proceso resolutorio. En el cuarto momento se ubica al docente como protagonista, debido a que debe lograr, a través de su 
intervención, formalizar los conocimientos que se pretendían propiciar con el problema, utilizando la resolución lograda por los alumnos (MEP, 2012).

\section{Segunda etapa: la movilización y aplicación de los conocimientos}

Su intención es abrir el espacio para que el alumnado ponga en práctica los conocimientos y habilidades adquiridas. En esta etapa se permite ampliar la gama de actividades para el uso de las destrezas en nuevos escenarios, tanto matemáticos como en contextos reales y de preferencia con diversidad en los niveles de complejidad de las tareas matemáticas (MEP, 2012; Ruiz, 2018).

Esta segunda etapa se puede asociar con las prácticas que tradicionalmente se han usado en la enseñanza de las Matemáticas, pero con la salvedad de no ser extremadamente extensas, con ejercicios repetitivos sin retos cognitivos importantes. Por tanto, no es una lista de ejercicios que persiguen solo un propósito, corresponde a situaciones que obliguen a los estudiantes a utilizar los conocimientos en nuevas circunstancias, propiciando espacios para la conexión entre la realidad, los contenidos de varias áreas matemáticas y disciplinares, lo que también implica el uso de diversas representaciones de un mismo objeto; la conexión y los otros procesos matemáticos que implican acciones cognitivas superiores que se hacen presentes al resolver problemas (Ruiz, 2018).

Es necesario hacer una aclaración sobre la resolución de problemas como noción y su perspectiva en los programas de Matemáticas (MEP, 2012) y en la política curricular (MEP, 2015).

Primero, se abordará la perspectiva desde los programas de Matemáticas, en la fundamentación teórica se proponen tres maneras: eje curricular, estrategia metodológica y proceso matemático (MEP, 2012). Para efectos de este apartado se expondrá el significado que tiene como eje curricular y como proceso matemático, sin embargo, no se abordará como estrategia metodológica porque fue presentado en la sección anterior.

El MEP (2012) adopta cinco ejes disciplinares que se resumen en el siguiente párrafo:

La resolución de problemas como estrategia metodológica principal, la contextualización activa como un componente pedagógico especial, el uso inteligente y visionario de tecnologías digitales, la potenciación de actitudes y creencias positivas en torno a las Matemáticas, finalmente, el uso de la historia de las Matemáticas (p. 17).

El primero de ellos, "resolución de problemas como estrategia metodológica principal" se puede considerar uno de los ejes más importantes, permea las acciones en el aula y es ejecutado mediante dos etapas como se expuso previamente.

Por otra parte, como proceso matemático se denomina "Plantear y resolver problemas" su significado corresponde a dos acciones: planteamiento de problemas y estrategias de solución de problemas. Respecto al planteamiento, se enfatizan los contextos reales como escenarios ideales para propiciar habilidades como identificar, formular, diseñar y desarrollar, mientras que contrastar y resolver, se asocian con estrategias, planes, modelos y métodos de resolución, así como la selección, el control y la evaluación de ellos, al enfrentar una tarea matemática (MEP, 2012).

Respecto a la política curricular, en la dimensión "maneras de pensar", se enuncian cinco habilidades, la citada como "resolución de problemas" no se evidenciará en el desarrollo de este documento, porque se asume como estrategia metodológica principal, por tanto, es el medio para la adquisición de conocimientos.

Sobre las dimensiones se ampliará en la sección 1.3, después de ejemplarizar una clase con la propuesta metodológica del MEP (2012), con el propósito de ofrecer los insumos necesarios para establecer la relación entre los programas de Matemáticas y la política curricular vigente. 


\section{Ejemplo de una clase mediante la resolución de problemas}

A continuación, se plantean las ideas básicas de una clase de Matemáticas usando la resolución de problemas, con el objetivo de mostrar cómo se puede desarrollar la primera etapa a través de los cuatro momentos en un espacio de dos lecciones.

Es necesario antes de presentar el problema, ubicarlo en décimo año, para propiciar la siguiente habilidad específica "determinar qué figuras se obtienen mediante secciones planas de una esfera y características métricas de ella" (MEP, 2012, p. 393), donde los conocimientos involucrados son: la esfera, el radio y el diámetro. Además, es importante indicar que el tiempo para que los estudiantes den una solución, puede ser de 15 a 20 minutos.

\section{Propuesta de un problema}

En el siguiente recuadro se presenta el problema que será empleado para desarrollar la estrategia metodológica en cuatro momentos.

\section{Tazón para ensalada}

La empresa Buen Comer de Costa Rica, fabrica tazones de diversos tamaños, que tienen formas como la siguiente:

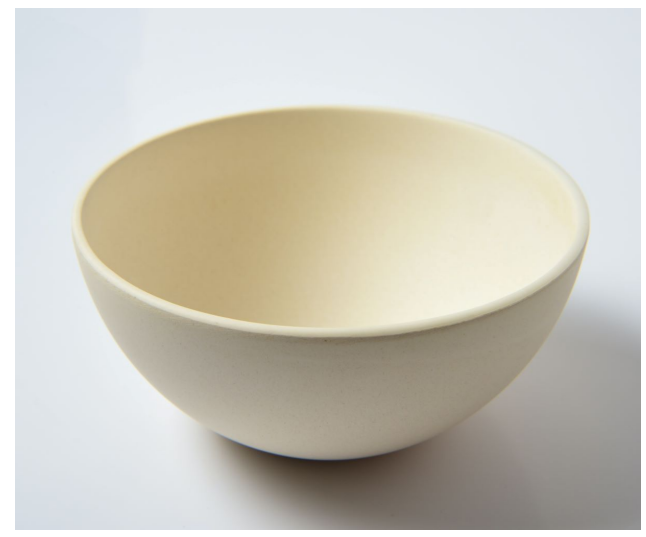

Se necesita construir una caja, para empacar el tazón y evitar que se fracture antes de llegar a las manos del cliente. El tazón tiene las siguientes dimensiones, en la parte superior un diámetro $26 \mathrm{~cm}$ incluyendo los bordes y en la parte inferior, se forma una circunferencia con $4 \mathrm{~cm}$ de radio. Puede usted ayudar al fabricante a determinar las dimensiones mínimas de la caja que se necesita para empacar el tazón.

Es importante aclarar, antes de presentar la solución del problema que, para efectos de la mediación pedagógica en el aula, se omiten elementos geométricos formales en el problema, de manera que se presente como una situación cotidiana, sin embargo, el docente debe estar atento a la importancia de aproximar la realidad a exactitud o rigurosidad matemática, por ejemplo, se debe asumir que el tazón tiene una forma semiesférica y que los planos involucrados son paralelos. Además, que la caja es un paralelepípedo y que las dimensiones deben ser una aproximación de la longitud real, por tanto, se debe determinar el valor entero mínimo en centímetros de la altura del interior de la caja, así como de su base.

A continuación, se presenta una posible solución, con el objetivo de poder articular los siguientes tres momentos para la organización de la lección y las dimensiones de la política curricular.

Se espera que los estudiantes comiencen por representar gráficamente la situación planteada, a través de dibujos hechos a mano o mediante algún software graficador que pueden acceder en línea con sus 
teléfonos inteligentes. Además, pueden colocar la información extraída del problema en la representación. Después de representar gráficamente la situación, que implica recordar que cualquier radio

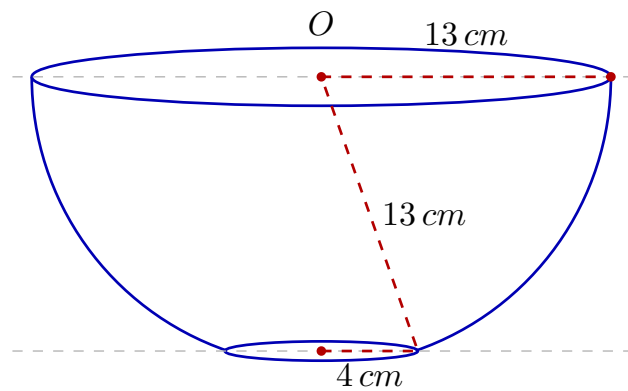

(a) Datos preliminares brindados en el problema.

Figura 1.2: Representación de la situación planteada en el problema “Tazón para ensalada". Fuente: Elaboración propia.

de la circunferencia mide $13 \mathrm{~cm}$, bastará con realizar los cálculos correspondientes para determinar la longitud de $x$ :

$$
\begin{aligned}
& x=\sqrt{13^{2}-4^{2}} \\
& x=\sqrt{153} \\
& x \approx 12,37 \mathrm{~cm}
\end{aligned}
$$

Por lo tanto, la respuesta corresponde a indicar que el valor entero mínimo en centímetros de la altura del interior de la caja que se necesita para empacar el tazón es de $13 \mathrm{~cm}$. Y esto implica que la caja debe tener las siguientes dimensiones aproximadamente, largo y ancho de $26 \mathrm{~cm}$ con una altura de $13 \mathrm{~cm}$.

\section{Trabajo estudiantil independiente}

Durante los 15 o 20 minutos que se asignarán para la resolución del problema en subgrupos, los estudiantes discutirán sobre cómo representar el problema. Podrán utilizar papel, lápiz y otros recurrir a sus dispositivos móviles. Otra opción, es que el docente haya solicitado material tangible como una bola de estereofón para simular la situación o haya llevado un recipiente con las características descritas, para representar el tazón. Además, comenzarán a realizar cálculos y aproximaciones, antes de establecer la estrategia de resolución.

Durante este espacio, utilizarán sus conocimientos matemáticos previos entre ellos: circunferencia, radio, diámetro, teorema de Pitágoras, triángulo rectángulo, rectángulo, cuadrado, entre otros. Además, realizarán un intercambio de ideas respecto a las siguientes interrogantes ¿cuál será el ancho mínimo de la caja? ¿cuál será el largo mínimo de la caja? y ¿cuál será la mejor forma de hallar la respuesta?

Se espera que, en este espacio, algunos de los subgrupos logren establecer el largo y ancho de la base de la caja y que algunos logren aproximar la altura mínima de la caja en $13 \mathrm{~cm}$. Puede ser que los restantes subgrupos, no consigan una solución satisfactoria, pero los errores cometidos pueden ser empleados para realimentar la siguiente etapa (Rico, 1998; Ruano, Socas y Palarea, 2008).

De acuerdo con el MEP (2012), en este momento de la clase, el docente debe supervisar los subgrupos para identificar dificultades durante el proceso de resolución y ofrecer preguntas generadoras que permitan salir del "estancamiento", entre ellas: ¿cuántos radios hay en una esfera? ¿cómo se pueden representar? ¿cómo se puede representar la información que presenta el problema gráficamente?, claro está, los cuestionamientos se deben plantear en función de los obstáculos que presenten los alumnos. 


\section{Discusión interactiva y comunicativa}

Respecto a este momento, el docente puede solicitar que se ubiquen en semicírculo, todos mirando hacia la pizarra e invitar a un subgrupo a exponer su estrategia de solución.

El docente puede haber seleccionado una estrategia novedosa, errónea o parcialmente correcta, eso dependerá de los propósitos didácticos del educador y de las particularidades del grupo. En la pizarra, se deben consignar los procesos de resolución por parte de los estudiantes, mientras que el docente, registra las opiniones, comentarios, preguntas e intervenciones de los alumnos sobre las anotaciones de la solución.

\section{Cierre y clausura}

Durante este último momento, el docente debe retomar el proceso de resolución del problema realizado por los estudiantes. Además, sistematizar algunos elementos teóricos sobre la esfera y sus propiedades, pero utilizando el problema empleado y la solución. En los aspectos por formalizar pueden estar las siguientes ideas:

La intersección de un plano y una esfera siempre es un círculo, cuando la intersección contiene al diámetro, se ha trazado el círculo máximo. Si se tiene un plano que pasa por el centro de la esfera, el radio del círculo contenido en el plano, es el mismo que el de la esfera. Se puede usar el recurso disponible en el enlace, para ampliar el tema (https : //recursoslibres. reformamatematica. net/ esfera/) y fortalecer la habilidad específica propiciada con el problema del tazón.

Después del cierre, con una síntesis como la anterior, se puede introducir un repaso del uso del teorema de Pitágoras, como un recurso para determinar la longitud faltante.

A continuación, la política curricular será descrita mediante sus dimensiones, de manera que se pueda articular con la mediación pedagógica expuesta hasta el momento.

\subsection{Dimensiones de la política curricular}

Para comprender la intención de la política curricular, aprobada por el CSE en el año 2016, se puede estudiar la figura 1.3 que se muestra seguidamente, la cual es fundamental debido a que corresponde a la aspiración de persona que debe ser modelada a través del sistema educativo costarricense.

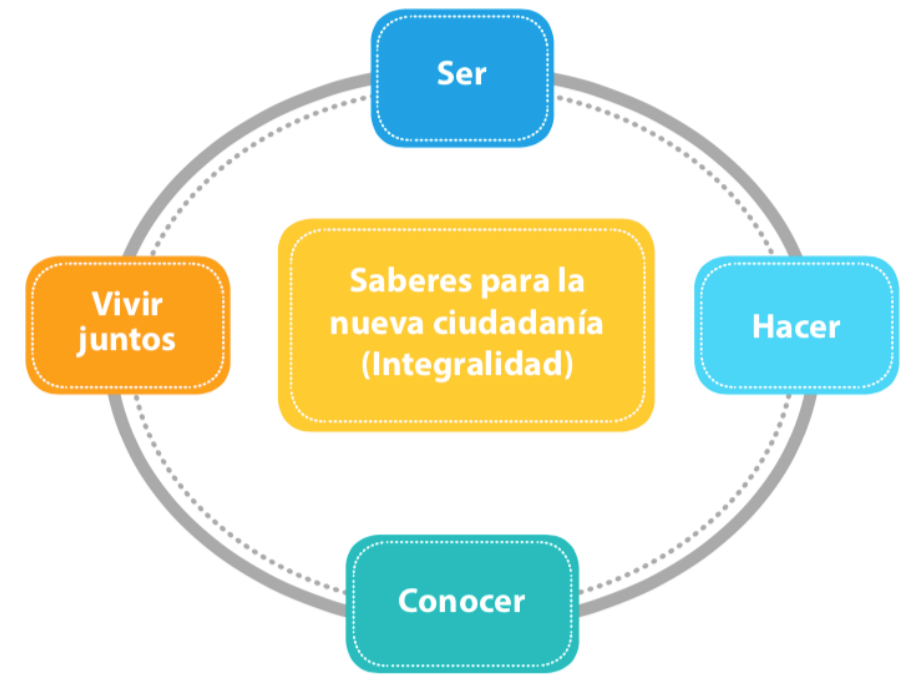

Figura 1.3: Esquema de los saberes para la nueva ciudadanía.

Fuente: MEP (2015, p. 32)

Se propone que a través de cuatro dimensiones, que se asocian con el ser, el conocer o saber, el convivir y el hacer, se puede favorecer el desarrollo de la persona de una manera integral, porque el estudiante a través de las oportunidades para adquirir conocimientos, debe lograr desarrollar su ser, porque hace 
y convive de manera simultánea (MEP, 2015).

Las dimensiones de la política curricular corresponden a las líneas estratégicas que deben orientar el quehacer en las aulas, como las aspiraciones máximas a seguir en todas las asignaturas del sistema educativo nacional. Sin embargo, no deben ser líneas conductoras, inflexibles y que, desde la generalidad, se intenten plasmar como herramientas para el planeamiento de aula.

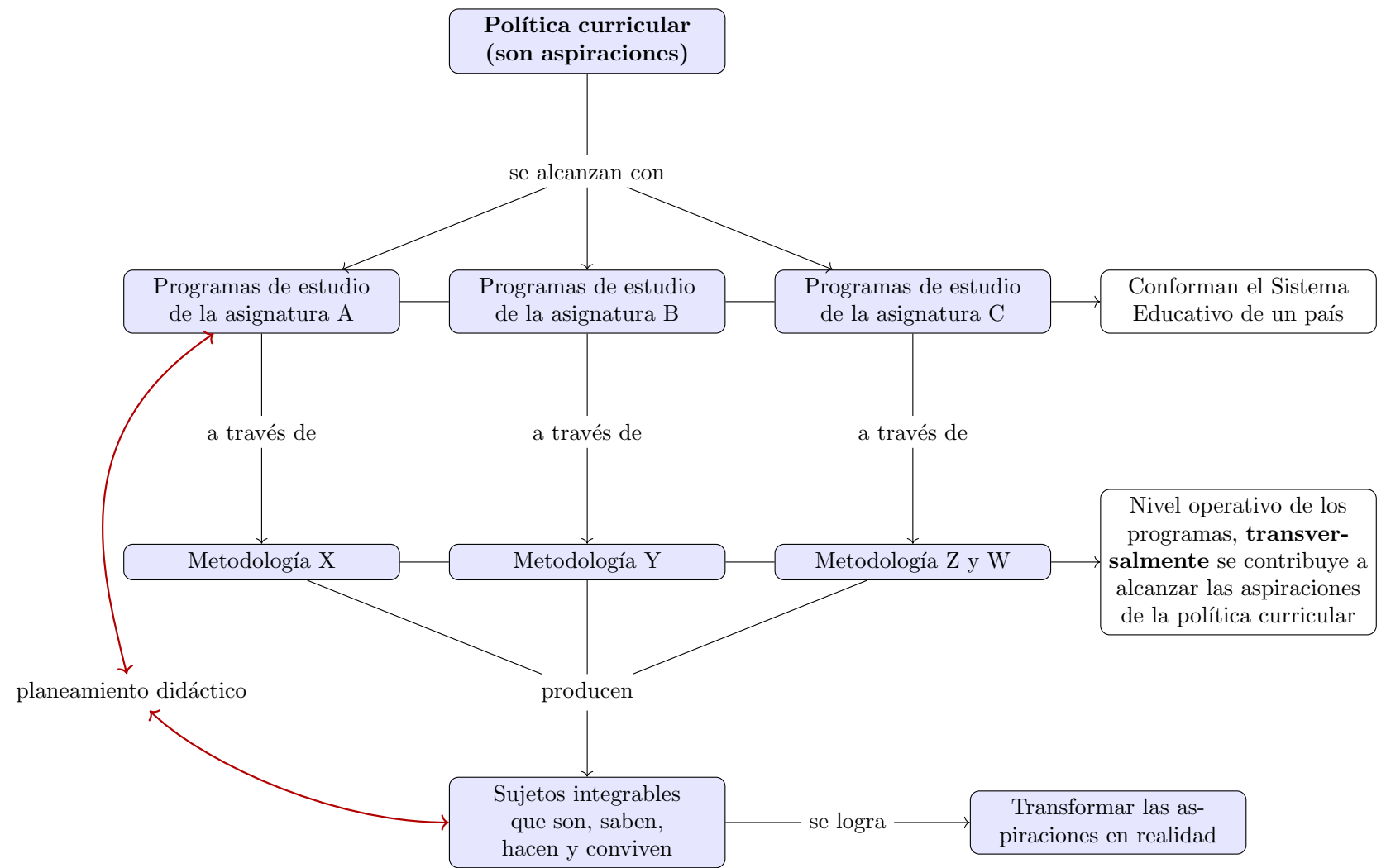

Figura 1.4: Interacción entre la política curricular y el sistema educativo.

Fuente: Elaboración propia.

En la figura 1.4 es importante señalar que la política curricular no es operacional por sí misma, es a través de los programas de estudio que se propician actividades en las aulas para alcanzar las aspiraciones que están plasmadas en ella. De acuerdo con Bongco y David (2020) comprender las aspiraciones de la política curricular es de suma importancia cuando se lleva a cabo la implementación de los programas oficiales de cualquier asignatura, pero eso no interfiere con la ejecución individual de cada programa.

Con el objetivo de favorecer la comprensión de la política curricular, se exponen a continuación las dimensiones que la conforman.

\section{Dimensión 1: Maneras de pensar}

En esta dimensión, se aspira a propiciar diversas habilidades de pensamiento, como parte del conocer o saber y señalan cinco maneras para lograrlo: pensamiento sistémico, pensamiento crítico, aprender a aprender, resolución de problemas y por último creatividad e innovación.

Una forma de acceder al conocimiento es propiciando el pensamiento sistémico, esto consiste en proponer situaciones de aprendizaje que permita visualizar "el todo y sus partes" (MEP, 2015, p.33), así como visualizar las conexiones entre la realidad o un contexto determinado. La visión incluida por el MEP (2015), es minimalista y limitado por tres indicadores, se restringe a esa visión de parte-todo.

Sin embargo, es importante señalar que su fundamento teórico es muy amplio porque implica "conexión, totalidad y relación" (Giraldo, 2016, p. 280). Se debe asumir que cualquier relación educativa y 
social implica comunicación y cultura, como un ente cuyas interrelaciones funcionan como organismos vivos. Por tanto, el pensamiento sistémico transciende el todo y sus partes, para permitir tratar un tema de interés escolar, como una unidad con múltiples interacciones entre sus componentes, sin separar en pequeñas partes las secciones que lo articulan.

Respecto al pensamiento crítico, se asocia con la claridad, la exactitud, la precisión, la relevancia, la profundidad y la priorización de la información, así como su manejo, debido a que se asumen como parte de las estructuras cognitivas. Si embargo, dentro de los indicadores no se establece ninguna acción de juzgamiento sobre situaciones del contexto o actividades de aprendizaje, únicamente se aspira a identificar pro y contras en el punto de vista propio y de otros (MEP, 2015).

Aprender a aprender, es otra manera de pensar que se quiere privilegiar, se asocia con la resolución de problemas y la autorregulación del aprendizaje mediante el conocimiento y su organización. En esta dimensión se considera, la resolución de problemas como una actividad que permite aprendizaje, desde la perspectiva de un escenario, el problema como casos por resolver. No se asocia con la otra dimensión de la política curricular o con la estrategia metodológica como se asume en los programas de Matemáticas (MEP, 2012; MEP, 2015).

Otro componente de esta dimensión, es la resolución de problemas, desde la política curricular es la "Habilidad de plantear y analizar problemas para generar alternativas de soluciones eficaces y viables" (MEP, 2015, p. 34), se puede establecer que esta habilidad aspira a la generación de soluciones por parte del estudiantado al enfrentar situaciones problemáticas, sin embargo, esto puede generar confusión respecto a los programas de Matemáticas, los cuales asumen la resolución de problemas de tres maneras diferentes: la primera como eje curricular, la segunda como estrategia metodológica y la tercera como proceso matemático, las tres como objetos individuales (MEP, 2012).

Respecto al último componente de la dimensión, se encuentra la creatividad e innovación, de acuerdo con el MEP (2015) corresponden a las acciones que conllevan a ideas originales y de utilidad como respuesta al contexto, de manera que se conviertan en alternativas novedosas.

Aunque esta dimensión, corresponde a la de mayor peso y se evidencia a través de un número mayor de componentes, se puede observar como ellas son aspiraciones. Corresponden al ideal que se pretende generar, pero ¿cómo hacerlo?, la respuesta se encuentra en el sistema educativo, a nivel operativo a través de cada asignatura y la ejecución de los programas oficiales, como se confirmará más adelante.

\section{Dimensión 2: Formas de vivir en el mundo}

Respecto al ser, en esta dimensión se proponen habilidades que son consideradas como necesarias para que la persona estudiante se desenvuelva en el ámbito personal, se incluyó la ciudadanía tanto global como local e ideas para enfrentar las responsabilidades tanto personales como sociales, lo que concuerda con las ideas de Morin $(1999 ; 2002)$, de acuerdo con el autor, se debe luchar contra la pérdida del derecho al conocimiento, porque esto va en detrimento de la calidad de vida y de la convivencia humana.

Al respeto Morin (2002) señala que el "debilitamiento de una percepción global conduce al debilitamiento del sentido de la responsabilidad" (p.8), por tanto, la inclusión de habilidades sobre la ciudadanía permite adquirir información de diversas culturas para favorecer la convivencia, la empatía y la tolerancia; con esto, se visibilizan las responsabilidades que cada sujeto tiene como parte de la sociedad. El otro componente de esta dimensión se asocia con un estilo de vida saludable y la proyección hacia el futuro, considerando un proyecto personal de vida, entre ellas una ocupación.

\section{Dimensión 3: Formas de relacionarse con los otros}

Respecto a la convivencia, las habilidades de esta dimensión son claras y concisas: colaboración y comunicación. Ambas, son indisolubles, implican trabajo en equipo donde los procesos de producción, 
comprensión e intercambio de mensajes corresponden a la herramienta fundamental (MEP, 2015).

Es importante, destacar que los programas de Matemáticas, incluyen el proceso matemático comunicar, como el intercambio de ideas relacionadas con objetos matemáticos de diversas maneras: oral, escrita o visual, en lenguaje cotidiano, formal, simbólico y técnico (Alsina y Coronata, 2015; MEP, 2012; OCDE, 2017; Ruiz, 2018).

\section{Dimensión 4: Herramientas para integrarse al mundo}

El hacer, es considerado en esta dimensión desde dos perspectivas: apropiación de las tecnologías digitales y el manejo de la información. La primera habilidad sugiere que se debe permitir la creación de elementos digitales para compartir y la segunda, propiciar la capacidad de acceder de manera crítica a la información con propósitos claros de forma efectiva (MEP, 2015).

Después de describir brevemente, la mediación pedagógica que plantean los programas de Matemáticas y las dimensiones que componen la política curricular, se hace necesario establecer la relación existente.

\subsection{Relación entre los programas de matemáticas y las dimensiones de la política curricular}

A continuación, se expondrán de nuevo los momentos para la organización de las lecciones de Matemáticas para la primera etapa y se analizará en cada espacio la presencia de las dimensiones de la política curricular para establecer la relación existente.

\section{Propuesta de un problema}

El tazón para ensalada corresponde a un problema contextualizado, donde el contexto se puede clasificar como activo, debido a que es indispensable para la solución de él (Ruiz, 2018). Esto permite, a la persona estudiante, enfrentar un reto matemático que se asocia con su realidad, favoreciendo la dimensión 1: maneras de pensar, porque activa el pensamiento sistémico al ofrecer a través de un trabajo escolar, en este caso un problema, abordar la situación y algunos de sus componentes entre ellos: el tazón para ensaladas de uso doméstico (que se asocia con un estilo de vida saludable), nociones matemáticas como: esfera, radio, sección plana, teorema de Pitágoras y las característica de una caja en forma de paralelepípedo, todos ellos poseen múltiples interacciones y trascienden "el todo y sus partes".

\section{Trabajo estudiantil independiente}

Durante este momento de la clase se ofrece el espacio para potenciar el saber, el ser, el convivir y el hacer, porque las cuatro dimensiones de manera sinérgica se hacen presentes y casi todas sus habilidades.

Durante los minutos asignados para la resolución del problema, los estudiantes deberán mantener una actitud colaborativa y de continua comunicación (dimensión 3), esto debido a que se debe crear una estrategia para encontrar la respuesta, proceso que favorece la creatividad y la innovación (dimensión 1). Este espacio implica que los estudiantes experimentaran discusiones sobre cómo representar el problema, tal vez al hacer un dibujo del tazón en papel, por tanto, se hace presente el pensamiento crítico (dimensión 1). En el caso de optar por una representación digital del problema (ver Figura 1.2), se está haciendo uso de las tecnologías digitales y el manejo de la información (dimensión 4).

La dimensión 2: Formas de vivir en el mundo, se hace presente de manera implícita, pero transversal durante todo el trabajo realizado, porque la persona estudiante debe autorregular su ser, favoreciendo la convivencia, aplicando la empatía y la tolerancia, mientras se construye una solución conjunta para determinar la altura mínima de la caja. 
Es importante señalar que el intercambio de preguntas sobre la altura, largo y ancho mínimo de la caja y la mejor estrategia para hallar la solución, permiten aprender a aprender (dimensión 1), debido a la actividad reflexiva que involucra.

\section{Discusión interactiva y comunicativa}

En este espacio de discusión colectiva, donde un subgrupo muestra su estrategia de solución se vuelve hacer presente la dimensión 1 en sus múltiples maneras a través de la criticidad, la interrelación y la autorregulación. Ellas surgen en el intercambio de ideas que se genera en el semicírculo dirigido por el docente, donde este último sistematiza una solución aceptada por el grupo completo. Además, la dimensión 2, estará siempre presente como la forma de relacionarse con otros, demostrando respeto y tolerancia durante la exposición.

\section{Cierre y clausura}

Este momento de la clase, es responsabilidad completa del docente (MEP, 2012; Zumbado-Castro, 2019), sin embargo, su interacción con la solución planteada por los estudiantes y el registro de las intervenciones de los alumnos, puede propiciar el espacio para favorecer una sana convivencia (dimensión 3) y crear sólidos cimientos para el saber que se perseguía con las habilidades específicas de Matemáticas, en este caso determinar qué figuras se obtienen mediante secciones planas de una esfera y características métricas de ella. A modo de cierre de este apartado, se debe enfatizar que la política curricular como conjunto de aspiraciones se puede concretar mediante la operacionalización de los programas de cada asignatura desde la particularidad, como en este caso mediante una clase de matemáticas de acuerdo con la metodología de resolución de problemas. Para lograr lo anterior, los docentes deben conocer la política curricular para potencializarla a través de su trabajo en el aula, pero se debe tener claro que no es conveniente considerarla como componente del planeamiento didáctico.

En la figura 1.5, se hace una síntesis de la relación entre la estrategia metodológica plantea en los programas de Matemáticas (MEP, 2012) y las dimensiones de la política curricular (MEP, 2015).

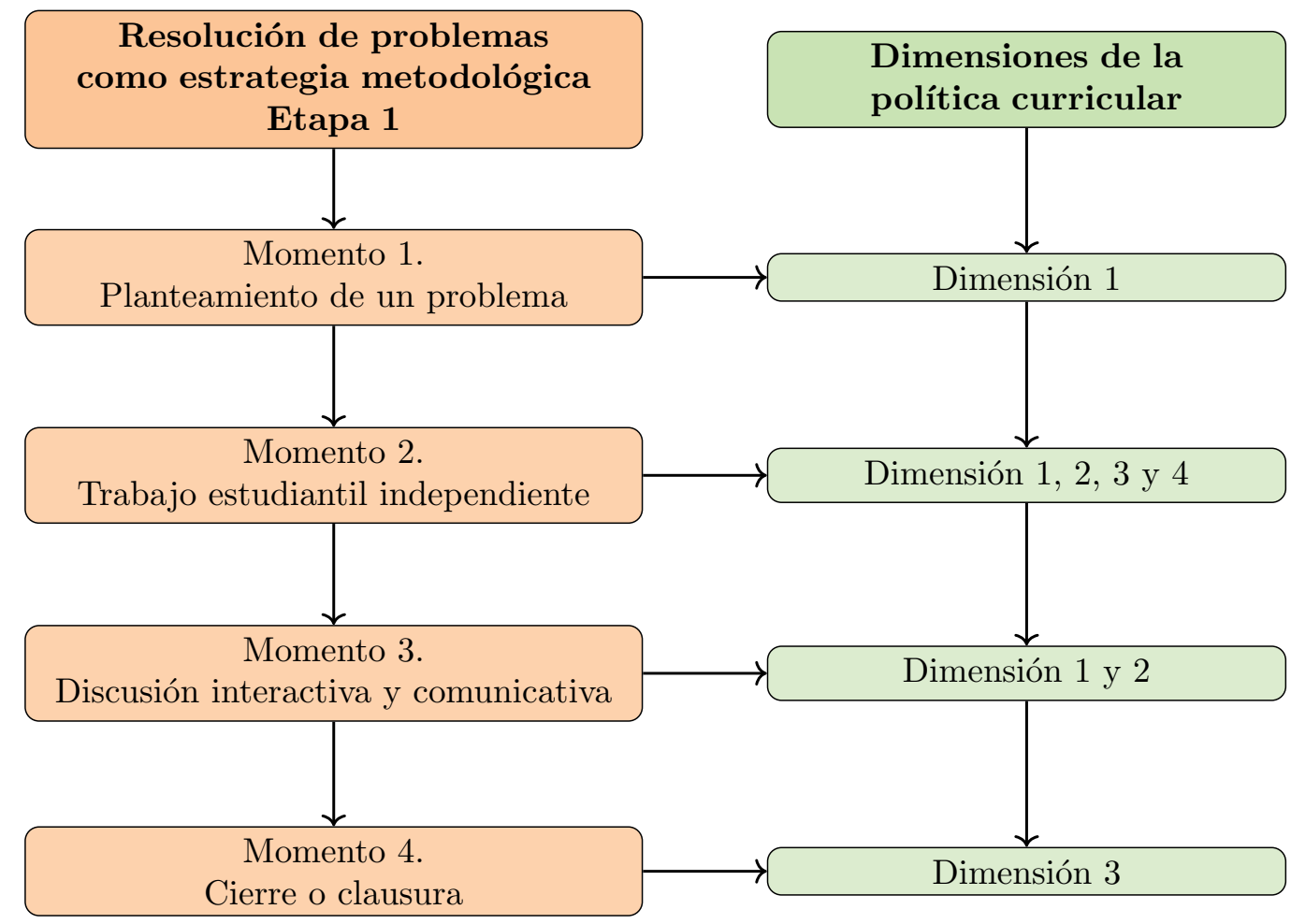

Figura 1.5: Relación entre la resolución de problemas como estrategia metodológica y la manera que se propician las habilidades de las dimensiones de la política curricular.

Fuente: Elaboración propia. 


\subsection{Consideraciones finales}

De acuerdo con la información suministrada sobre la estrategia metodológica de resolución de problemas propuesta por el MEP (2012) para la organización de las clases de Matemáticas, se puede afirmar que desde su composición teórica y operativa las lecciones que cumplan los cuatro momentos lograrán establecer una relación con la política curricular "Educar para una Nueva Ciudadanía" (MEP, 2015), porque ofrece el espacio para que las cuatro dimensiones actúen de manera sinérgica en el salón, por tanto, potencian cada una de las habilidades que componen las aspiraciones del sistema educativo costarricense, porque la resolución de problemas propicia de manera natural su interacción.

La relación existente, facilita el alcance de las aspiraciones que se plasman sobre nuestro sistema educativo, que pretende egresar personas integrales. Por tanto, es de suma importancia, que el docente de matemáticas comprenda las dimensiones de la política curricular y su relación con la resolución de problemas como estrategia metodológica principal (MEP, 2012) para potencializarla mediante los momentos de la clase.

Se considera poco afortunada la decisión de hacer operativa la política curricular dentro del planeamiento de aula y la evaluación de los aprendizajes de manera homogénea, rígida y mecanizada a través de tablas, indicadores y rasgos, debido a que se concuerda con Bongco y David (2020), en que la política curricular son aspiraciones que deben ser comprendidas por la persona docente, sin embargo, no deben interferir en la ejecución de las asignaturas, por sus particularidades. Cada una de las asignaturas y sus respectivos programas, han sido diseñados desde la perspectiva de su didáctica específica, el caso de Matemáticas es un ejemplo, por tanto, no es apropiado enfatizar las habilidades de las dimensiones de la política curricular, como articuladora de los planeamientos didácticos, debido a que implica violentar la malla curricular elaborada por especialistas en la asignatura y desvirtuar la perspectiva con la que se concibieron.

En el caso de Matemáticas, se desvirtúa la competencia matemática que se persigue, lo que implica, también infringir los elementos aprobados por el CSE, cuando dio el visto bueno a los programas.

Por tanto, aunque se ha mostrado una relación entre los programas de matemáticas, su estrategia metodológica y la política curricular, la última no debe interferir en el plan de clase sino potenciarla.

Conclusiones y recomendaciones

Respecto a las preguntas iniciales, la primera planteaba ¿cuál es la relación entre los programas y la política curricular? se puede concluir que existe una relación entre ambos, de manera que al desarrollar una clase de Matemáticas mediante la estrategia metodológica de resolución de problemas se propicia el espacio para alcanzar las aspiraciones que tiene el sistema educativo costarricense y que no es lo más conveniente emplear la política curricular para articular los planeamientos de aula.

La segunda pregunta planteaba ¿cuáles implicaciones tiene para el docente de Matemáticas? Al respecto se puede concluir que es necesario que el docente de matemáticas comprenda en profundidad dos cosas: la estrategia metodológica de resolución de problemas según la propuesta del MEP (2012) y la política curricular, debido a que la aplicación de la estrategia metodológica propicia un trabajo de aula que favorece las dimensiones de la política curricular.

Finalmente, para favorecer las habilidades relacionadas con las dimensiones de la política curricular, desde el área de Matemáticas específicamente, se sugiere:

1. Emplear la estrategia metodológica de resolución de problemas, experimentando los cuatro momentos, debido a que se crea un escenario dentro del aula que favorece de manera simultánea y natural las cuatro dimensiones de la política curricular.

2. Usar problemas contextualizados, la mayor cantidad de veces posible en el aula, porque esto favorece la capacidad de razonar y argumentar; Además, de establecer conexiones entre diversas 
áreas del conocimiento como: Biología (crecimiento de bacterias), Economía (interés compuesto) o Administración (media ponderada).

3. Para promover la Dimensión 1: Maneras de pensar, se sugiere el planteo de problemas que impliquen situaciones retadoras que inviten a la investigación, creación de modelos o soluciones alternativas, como por ejemplo: problemas vinculados con la arquitectura y el uso del triángulo en ella, debido a que él posee la capacidad de sostener y distribuir la fuerza que se le ejerce y por tanto, no se deformar.

4. La Dimensión 2: Formas de vivir en el mundo, puede ser favorecida de manera más significativa, si se seleccionan problemas que impliquen datos estadísticos, debido a que propician escenarios de reflexión si se vinculan con temas sensibles de la sociedad, por ejemplo, se pueden utilizar los recursos del Instituto Nacional de Estadística y Censo (INEC), porque ofrecen información sobre diversos ámbitos, entre ellos: población, empleo, género y economía, lo que permite conocer con datos actuales la realidad del país respecto a la diversidad poblacional, el desempleo y sus implicaciones, la inequidad por género respecto al empleo o la remuneración, entre otros tema; Con esto se otorga al estudiante la posibilidad de aprender a ser un ciudadano que comprende su entorno y asuma en su momento las responsabilidades que exige la Sociedad.

5. Aunque se ha manifestado la transversalidad de la Dimensión 3: Formas de relacionarse con los otros, al emplear la estrategia metodológica de resolución de problemas, parece conveniente también dar una idea puntual. Si se plantea un problema de Geometría que se relacione con el arte, por ejemplo, los teselados de Escher, se puede proponer un proyecto en coordinación con la asignatura de Artes Plásticas para favorecer un trabajo en equipo y las habilidades de colaboración y comunicación, porque ellas son indispensables para lograr de manera exitosa el producto artístico que utiliza transformaciones isométricas como: la rotación, la traslación y la simetría.

6. Algunos problemas de Relaciones y álgebra, que implique modelización favorecen la Dimensión 4: Herramientas para integrarse al mundo, debido a que a través de aplicaciones y softwares se puede simular la realidad, por tanto, se generan las condiciones para acceder de manera crítica a la información y responder a un propósito claro, en este caso identificar un modelo.

\section{Bibliografía}

[1] Alsina, A. y Coronata, C. (2015). Los procesos matemáticos en las prácticas docentes: diseño, construcción y validación de un instrumento de evaluación. Edma 0-6: Educación Matemática en la Infancia, 3(2), 23-36.

[2] Barrantes, H. (2006). Matemáticas y razonamiento plausible. Cuadernos de Investigación y Formación en Educación Matemática. 1(1), 1-9.

[3] Bongco, R \& David, A. (2020). Filipino teachers' experiences as curriculum policy implementers in the evolving K to 12 landscape. Issues in Educational Research, 30(1), 19 ?34. Recuperado en http://web.a.ebscohost.com.cidreb.uned.ac.cr/ehost/pdfviewer/ pdfviewer?vid=7\&sid=f6eee71f - e9a6 - 468a- 8 be6 -5 da 45 f8b7788\%40sessionmgr 4007

[4] Chavarría, S. (2010). La Política Educativa hacia el Siglo XXI: Propuesta de una Poli?tica de Estado. Ponencia preparada para el Tercer informe Estado de la Educación. San José: PEN

[5] Giraldo, L. (2016). El pensamiento sistémico y sus relaciones con el ámbito educativo: del paradigma lineal a la trama circular. Revista Filosofía UIS, 15(1), 279-312. Recuperado de https://revistas .uis.edu.co/index.php/revistafilosofiauis/article/view/ $6050 / 6314$ 
[6] Jiménez, R. (2014). Educación pública en Costa Rica: políticas, resultados y gasto. Análisis, 6 (Agosto). Recuperado de https : //www. academiaca.or.cr/wp-content/uploads/ 2017/09/Ronulfo. 2014-2.pdf

[7] Ministerio de Educación Pública [MEP]. (2015). Educar para una nueva ciudadanía: Fundamentación de la transformación curricular costarricense. Costa Rica: autor. Recuperado de http://www.idp.mep.go.cr/sites/all/files/idp_mep_go_cr/publicaciones/72016_educar_para_una_nueva_ciudadaniafinal.pdf

[8] Ministerio de Educación Pública [MEP]. (2012). Programas de estudio de Matemáticas para la Educación General Básica y el Ciclo Diversificado. San José, Costa Rica: autor. Recuperado de http://www.mep.go.cr/sites/default/files/programadeestudio/programas/ matematica.pdf

[9] Morin, E (1999). Los siete saberes para la educación del futuro. Paris, Francia: UNESCO. Recuperado de: http://edgarmorinmultiversidad .org/index. php/descarga-libro- los 7- saberes.html

[10] Morin, E (2002). La cabeza bien puesta, Buenos Aires, Argentina: Nueva Visión.

[11] Organización para la Cooperación y el Desarrollo Económico [OCDE]. (2017). Marco de Evaluación y de Análisis de PISA para el Desarrollo: Lectura, matemáticas y ciencias, Versión preliminar, Paris: OECD Publishing. Recuperado de https : //www. oecd .org/pisa/ aboutpisa/ebook $\% 20-\% 20$ PISA- D\%20FrameworkPRELIMINARY\%20versionSPANISH . pdf

[12] Rico, L. (1998). Errores y dificultades en el aprendizaje de las matemáticas. En J. Kilpatrick, P. Gómez y L. Rico. Educación Matemática. Errores y dificultades de los estudiantes. Resolución de problemas. Evaluación. Historia (pp. 69-104). Bogota: Grupo Editorial Iberoamérica. Recuperado de https://www. researchgate.net/publication/278009025 Educacion_Matematica_Errores_y_dificultades_de_los_estudiantes_Resolucion_ de_problemas_Evaluacion_Historia

[13] Ruano, R., Socas, M. y Palarea, M. (2008). Análisis y clasificación de errores cometidos por alumnos de secundaria en los procesos de sustitución formal, generalización y modelización en álgebra. Revista PNA 2(2),61-74. Recuperado de: http: //digibug . ugr . es / bitstream/10481/4441/1/Ruano2008Analisis.pdf

[14] Ruiz, A. (2018). Evaluación y pruebas nacionales para un currículo de Matemáticas que enfatiza capacidades superiores. Ciudad de México: CIAEM. Recuperado de https://www. angel ruizz.com/wp-content/uploads/2019/02/Angel-RuizEvaluacion-y-pruebas-2018.pdf

[15] Zumbado-Castro, M. (2019). Evidencias sobre algunas tendencias pedagógicas y didácticas presentes en los programas de estudio de matemáticas costarricense. Uniciencia, 33(2), 27-41. https://doi.org/10.15359/ru.33-2.3

[16] Zumbado, M., Espinoza, J., Espinoza, J., González, M. \& Ramírez, I. (2008). La Resolución de Problemas en la Enseñanza de las Matemáticas: una experiencia con la función exponencial, polígonos y Estadística (Tesis de licenciatura). Universidad Nacional, Heredia, Costa Rica. 